\title{
Aiming to Improve Readmissions Through InteGrated Hospital Transitions (AIRTIGHT): a Pragmatic Randomized Controlled Trial
}

\author{
Andrew McWilliams, MD, MPH ${ }^{7}$, Jason Roberge, $P h D^{7}$, William E. Anderson, $\mathrm{MS}^{7}$, \\ Charity G. Moore, PhD², Whitney Rossman, MS' , Stephanie Murphy, DO', Stephannie McCall, MD', \\ Ryan Brown, $\mathrm{MD}^{7}$, Shannon Carpenter, $\mathrm{MD}^{7}$, Scott Rissmiller, $\mathrm{MD}^{7}$, and Scott Furney, $\mathrm{MD}^{7}$ \\ ${ }^{1}$ Carolinas Health Care System, Charlotte, NC, USA; ${ }^{2}$ University of Pittsburgh, Pittsburgh, PA, USA.
}

\begin{abstract}
BACKGROUND: Despite years of intense focus, inpatient and observation readmission rates remain high and largely unchanged. Hospitals have little, robust evidence to guide the selection of interventions effective at reducing 30-day readmissions in real-world settings.
\end{abstract}

OBJECTIVE: To evaluate if implementation of recent recommendations for hospital transition programs is effective at reducing 30-day readmissions in a population discharged to home and at high-risk for readmission.

DESIGN: A non-blinded, pragmatic randomized controlled trial (Clinicaltrials.gov: NCT02763202) conducted at two hospitals in Charlotte, North Carolina. PATIENTS: A total of 1876 adult patients, under the care of a hospitalist, and at high risk for readmissions. INTERVENTION: Random allocation to a Transition Services (TS) program $(n=935)$ that bridges inpatient, outpatient, and home settings, providing patients virtual and in-person access to a dedicated multidisciplinary team for 30-days, or usual care $(n=941)$.

MAIN MEASURE: Thirty-day, unplanned, inpatient, or observation readmission rate.

KEY RESULTS: The 30-day readmission rate was $15.2 \%$ in the TS group and $16.3 \%$ in the usual care group (RR 0.93; 95\% [CI, 0.76 to 1.15$]$ ]; $P=0.52$ ). There were no significant differences in readmissions at 60 and 90 days or in 30-day Emergency Department visit rates. Patients, who were referred to TS and readmitted, had less Intensive Care Unit admissions $15.5 \%$ vs. $26.8 \%$ (RR $0.74 ; 95 \%$ [CI, 0.59 to 0.93]; $P=0.02$ ).

CONCLUSIONS: An intervention inclusive of contemporary recommendations does not reduce a high-risk population's 30-day readmission rate. The high crossover to usual care $(74.8 \%)$ reflects the challenge of non-participation that is ubiquitous in the real-world implementation of population health interventions.

TRIAL REGISTRY: ClinicalTrials.gov; registration ID number: NCT02763202, URL: https://clinicaltrials.gov/ ct2/show/NCT02763202

Received January 19, 2018

Revised May 23, 2018

Accepted July 18, 2018

Published online August 14, 2018
KEY WORDS: readmissions; population health; outcomes research; healthcare value; pragmatic research.

J Gen Intern Med 34(1):58-64

DOI: $10.1007 / \mathrm{s} 11606-018-4617-1$

(C) Society of General Internal Medicine 2018

\section{INTRODUCTION}

In 2012, the Affordable Care Act (ACA) established the Hospital Readmissions Reduction Program (HRRP), authorizing the Center for Medicare and Medicaid Services (CMS) to decrease payments to hospitals with excess readmissions. The intended consequence of HRRP is to change hospital system behavior, leading to improved population health outcomes. ${ }^{1}$ However, evidence of HRRP's effects on outcomes is inconsistent, and conclusions are limited by the observational nature of most studies. $^{1}$ From 2007 to 2015, readmission rates for HRRP-targeted conditions declined from 21.8 to $17.8 \%$, but non-targeted conditions also declined from 15.3 to $13.1 \% .^{2}$ During this same timeframe, observation stays experienced absolute increases of $2.1 \%$ and $1.7 \%$ for targeted and non-targeted conditions respectively. Though some analyses suggest a modest effect on readmissions from HRRP, others demonstrate an effect limited only to acute myocardial infarction (AMI). ${ }^{2-4}$

Regardless of HRRP's overall effects, the composite rate of inpatient and observation readmissions remains high and largely unchanged, while hospitals continue to be exposed to significant financial penalties. Despite years of attention, little, robust evidence exists to guide hospitals in selecting interventions effective at reducing 30-day readmissions in real-world settings. ${ }^{5-7}$ Current recommendations call for multifaceted interventions focused on patients at highest risk for readmission, while incorporating dedicated personnel to bridge inpatient, outpatient, and home delivery settings. ${ }^{7-11}$ To date, only two randomized controlled trials (RCTs) have evaluated interventions incorporating most of these recommendations, with one showing no effect and the other showing a 33\% relative reduction in readmissions. ${ }^{10,12}$ No RCTs have evaluated these interventions through a pragmatic trial. Pragmatic trials minimize exclusion criteria and use routinely collected outcomes to 
determine the effects of an intervention in the "real-world" setting compared to usual care, thus generating broadly applicable results. ${ }^{13,14}$

The primary objective of this study was to evaluate, in a pragmatic RCT, if implementation of the most recent recommendations for hospital transition programs is effective at reducing 30-day readmissions. Our healthcare system incorporated these recommendations into a comprehensive program called Transition Services (TS) ${ }^{15}$ We aimed to evaluate the hypothesis that referral to the TS program results in lower 30-day readmission rates compared to usual care in a population discharged to home and at high-risk for readmission.

\section{METHODS}

Aiming to Improve Readmissions Through InteGrated Hospital Transitions (AIRTIGHT) is a non-blinded, pragmatic RCT with patients allocated to receive either referral to TS or usual care. Details of the study design and intervention are published but described briefly here. ${ }^{16}$ This trial is pragmatic across all nine Pragmatic-Explanatory Continuum Indicator Summary 2 (PRECIS-2) domains. ${ }^{14,16}$

\section{Eligibility}

Patients were eligible if during the first $72 \mathrm{~h}$ of admission they were over age 18 , seen by a hospitalist, categorized as inpatient, and identified as high-risk for readmission using a risk scoring model. To guide readmission strategies, our healthcare system developed a risk model incorporating 71 patient-level clinical variables, resulting in daily risk scores between 0 and $1 .{ }^{16}$ Highrisk was defined by a score associated with a greater than $20 \%$ readmission rate among the reference population. Patients were excluded for primary residence more than 150 miles from the hospital; admitted from or discharged to hospice, skilled nursing facility (SNF), or jail; left the hospital against medical advice; presence of acute psychiatric illness or drug dependency; referred as part of AIRTIGHT in the previous 90 days; attended a similar heart failure transitions clinic at discharge; or active diagnosis of cancer or sickle cell disease. These latter groups were excluded because similar transition services were already in place. Since bridging interventions must begin prior to discharge, random allocation of referrals occurred within the first $72 \mathrm{~h}$ after admission; but for the purposes of outcomes analyses, exclusion criteria were applied again at discharge. This was necessary because TS was specifically designed to support the unique needs of patients discharged to home.

\section{Setting}

The TS program was evaluated for patients admitted to either a tertiary hospital or a smaller satellite hospital, both located within an integrated healthcare system in Charlotte, North Carolina, from February 2016 through January 2017.
Outcomes data were available from the system's 40 owned or managed hospitals.

\section{Trial Intervention and Control}

Control patients received usual care, which typically included recommendations for follow-up appointments with a patient's primary care physician (PCP), discharge summaries sent to PCPs by fax or the Electronic Medical Record (EMR), arrangement of home health services based on each patient's needs, and other outreach such as follow-up care management phone calls.

For the intervention group, a nurse navigator at each site attempted to contact referred patients prior to discharge with an invitation to participate in TS, provided an information pamphlet that introduced the TS program, and then followed hospital progress via the EMR. For 30 days following discharge to home, these patients had access to TS, which in addition to a free-standing clinic included (a) access to transition-dedicated internal medicine, pharmacist, paramedicine, behavioral health, and social work providers with scheduling frequency and provider type matched to patient needs; (b) hospital follow-up evaluation with a medical provider, either virtually in the patient's home facilitated by a paramedicine provider or in the transition clinic; (c) comprehensive medication reconciliation by a pharmacist; (d) at least weekly contact with a team member; and (f) coordinated transition to the next appropriate care location after 30 days. At a minimum to qualify as having participated, patients had an in-person or virtual visit with the TS medical provider. In keeping with the pragmatic, population health focus of this study, patients were not screened for interest in participation; rather, all who qualified for referral were included in analyses.

\section{Outcomes}

The primary outcome was 30-day, unplanned inpatient or observation readmission rate at any of 40 hospitals within the sponsoring healthcare system. We included observation admissions to create a more patient-centric outcome, because from a patient's perspective, any return to the hospital after discharge is viewed as a readmission. Secondary outcomes included separate 30-day inpatient or observation readmission rates, 30-day Emergency Department (ED) utilization, 60- and 90-day readmission rates (using the primary outcome definition), Intensive Care Unit (ICU) stay on readmission, 30-day readmission for subgroups, and length of stay (LOS) on index and readmission visits. The research team had no contact with participants and all outcomes were retrieved electronically from EMR data.

\section{Sample Size}

This study was designed to detect a $30 \%$ relative reduction in the readmission rate with the usual care group assumed to have a $20 \%$ 30-day readmission rate. ${ }^{6,17-19}$ To detect this reduction with $80 \%$ power, we calculated a total sample size estimate of $1230(\alpha=0.05)$ using a chi-square test for independence. 
Assuming $10 \%$ of those assigned to TS would not participate, we increased the sample size to $1520(n=760$ per arm $)$ to account for the anticipated decrease in effect size.

\section{Randomization}

Using permuted block randomization and block size of six that included both hospitals, patients were randomly allocated during their initial $72 \mathrm{~h}$ of admission in a 1:1 ratio to receive either referral to TS or usual care, using SAS software with future allocations concealed from the clinical team. ${ }^{20}$ Each weekday, an automated SAS program populated a TS referral list with newly eligible patients and emailed the list to both navigators. Given the intervention, it was not possible to blind patients or clinicians.

\section{Analysis}

The primary analysis followed the intention-to-treat (ITT) principle, such that patients were analyzed on the basis of the group to which they were initially randomized, after re-application of exclusion criteria at the time of hospital discharge (Fig. 1). In keeping with the pragmatic, population health focus of this study, patients were not screened for interest in participation; rather, the ITT analyses tested the effect of referral. Baseline comparisons of the two groups were made using the chi-square test for categorical variables and Student's $t$ test for age. The primary outcome, 30-day readmission rate, was compared between groups using the chi-square test. ${ }^{16}$ Results are presented as relative risks and $95 \%$ confidence intervals. The chi-square test was used to compare the two groups on secondary outcomes that were dichotomous, and the Wilcoxon rank sum test was used for LOS. Pre-specified subgroup analyses were conducted for patients with a primary diagnosis on index admission of congestive heart failure, pneumonia, and sepsis. We did not pre-specify analysis for AMI because many AMI patients are cared for by the cardiology service at the sponsoring institution and thus not eligible for study inclusion.
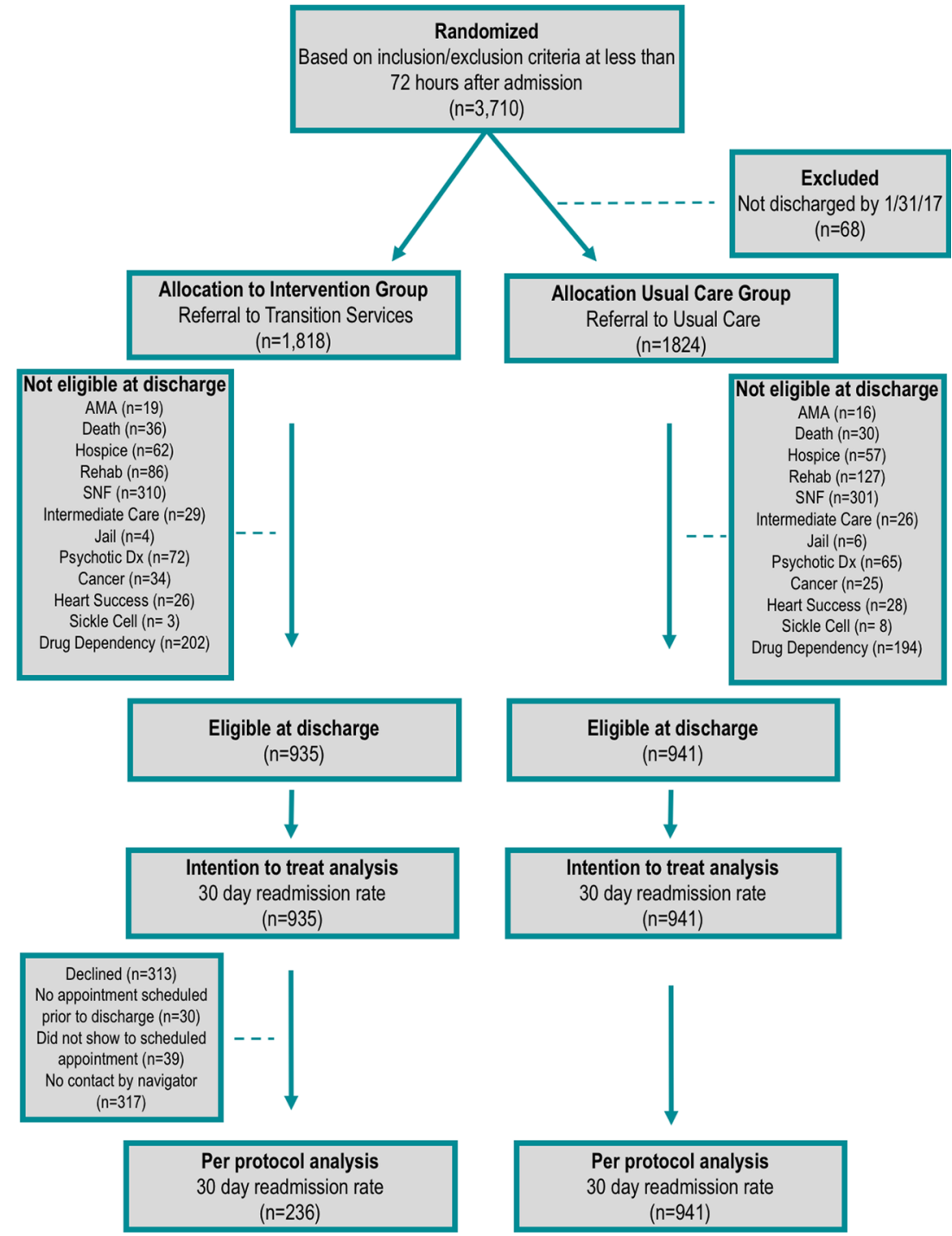

Figure 1 Study design and patient flow. 
Statistical analyses were performed using SAS Enterprise Guide $6.1{ }^{20}$ For all outcomes, we assume that if there were no visits in the EMR, the value for having a visit was null; therefore, there are no missing data for the primary or secondary outcome measures.

Given the magnitude of non-participation in the intervention group, we secondarily performed per-protocol (PP) and Complier Average Causal Effects (CACE) analyses to evaluate the effect of TS participation, in contrast to ITT analyses evaluating effectiveness of TS referral. The CACE analysis compared those who participated in TS to those in the control group who would have participated if given the opportunity; the underlying assumptions and framework have been previously published. ${ }^{21,22}$

When primary outcomes were available for 792 participants, a data and safety monitoring board (DSMB) met and provided recommendations based on planned interim efficacy and futility analyses, and a sample size reestimation using the overall readmission and dropout rates from the intervention group. At that time, the study did not meet the pre-specified efficacy or futility stopping criteria; however, the DSMB recommended targeting higher enrollment due to the high rate of non-compliance. The trial's steering committee and principal investigator decided, based on health system factors, to end the trial at the prespecified target.

\section{Ethics}

The trial was approved by the Carolinas HealthCare System Institutional Review Board and granted a waiver for patient consent (reference number 01-15-10E). The trial is registered with clinicaltrials.gov, NCT02763202.

\section{Role of Funding Source}

The study was funded by the sponsoring health system. No external funding or group(s) influenced the study design, analysis, or interpretation of results.

\section{RESULTS}

\section{Patients}

From February 2016 through January 2017, 3710 patients met inclusion criteria within $72 \mathrm{~h}$ of admission and 3642 were randomly allocated to referral to the intervention or usual care groups (68 were removed due to not being discharged by end of study). After reapplication of exclusion criteria at discharge, 1876 remained in the study and all were included in the ITT analysis (Fig. 1). The primary reasons for exclusion were being discharged to SNF (34.6\%) and meeting drug dependency criteria (22.4\%). Baseline characteristics were well balanced across groups (Table 1). Among those referred to TS, $25.2 \%$ participated in the program.
Table 1 Baseline Characteristics

\begin{tabular}{lll}
\hline \hline & $\begin{array}{l}\text { Transition } \\
\text { services } \\
(\boldsymbol{n}=\mathbf{9 3 5})\end{array}$ & $\begin{array}{l}\text { Usual } \\
\text { care } \\
(\boldsymbol{n}=\mathbf{9 4 1})\end{array}$ \\
\hline Male, $n(\%)$ & $457(48.9)$ & $447(47.5)$ \\
Hispanic, $n(\%)$ & $\begin{array}{l}n=869) \\
(n=883)\end{array}$ & $55(6.2)$ \\
Race, $n(\%)$ & $(n=916)$ & $(n=928)$ \\
American Indian/Alaska Native & $1(0.1)$ & $2(0.2)$ \\
Asian & $15(1.6)$ & $9(1.0)$ \\
Black & $360(39.3)$ & $373(40.2)$ \\
White & $463(50.6)$ & $481(51.8)$ \\
Multi & $2(0.2)$ & $2(0.2)$ \\
Other & $75(8.2)$ & $61(6.6)$ \\
Age, mean (SD) & $58.3(17.7)$ & $59.4(17.4)$ \\
Comorbidities, $n$ (\%) & $197(21.1)$ & $193(20.5)$ \\
Chronic obstructive pulmonary & $197)$ & \\
disease & $225(24.1)$ & $218(23.2)$ \\
Congestive heart failure & $359(38.4)$ & $363(38.6)$ \\
Diabetes & $112(12.0)$ & $107(11.4)$ \\
End-stage renal disease & $(n=924)$ & $(n=923)$ \\
Health Insurance, $n(\%)$ & $265(28.7)$ & $274(29.7)$ \\
Commercial/private & $134(14.5)$ & $129(14.0)$ \\
Medicaid & $396(42.9)$ & $405(43.9)$ \\
Medicare & $102(11.0)$ & $90(9.8)$ \\
Self pay & $27(2.9)$ & $25(2.7)$ \\
Other & $656(70.2)$ & $669(71.1)$ \\
Index admission at tertiary hospital & & \\
\hline
\end{tabular}

\section{Intervention Outcomes}

We did not detect a difference in 30-day readmission rates between the two groups $(15.2 \%$ for the group referred to TS and $16.3 \%$ for usual care; RR $0.93 ; 95 \%$ CI, 0.76 to $1.15 ; p=$ $0.52)$. Results were similar when examining only readmissions classified as inpatient (RR $0.98 ; 95 \% \mathrm{CI}, 0.77$ to $1.23 ; p=0.83$ ) and ED visit rates (RR 1.10; $95 \%$ CI, 0.95 to $1.28 ; p=0.20$ ). At 60 and 90-days, readmission rates across groups were similar (RR $0.97 ; 95 \%$ CI, 0.82 to $1.15 ; p=0.71$, and RR $0.96 ; 95 \% \mathrm{CI}$, 0.83 to $1.11 ; p=0.56$, respectively) (Table 2).

Among those readmitted, patients randomized to TS referral $(n=142)$ were less likely to have ICU admissions $15.5 \%$ vs. $26.8 \%$ (RR $0.74 ; 95 \%$ CI, 0.59 to $0.93 ; p=0.02$ ) compared to usual care $(n=153)$. On readmission visits, overall LOS was similar (median (IQR) 3.0 (4.0) days TS vs. 3.0 (4.0) days usual care; $p=0.52$ ) (Table 2).

For ITT subgroup analyses, referral to TS for patients with a primary diagnosis of sepsis $(n=139$ referred with a similar $26.6 \%$ participation rate) showed a reduction in 30-day readmission rates (RR $0.49 ; 95 \%$ CI, 0.24 to $0.97 ; p=0.03$ ), but not for congestive heart failure ( $n=41$; RR $1.17 ; 95 \%$ CI, 0.51 to $2.67 ; p=0.71$ ) or pneumonia ( $n=33$; RR $0.44 ; 95 \%$ CI, 0.12 to $1.65 ; p=0.26)$.

\section{Per-Protocol Outcomes}

When comparing TS patients who participated $(n=236,25.2 \%)$ to those who did not $(n=699)$, the participants were more likely to be African American, self-pay insurance status, or Medicaid (Appendix Table 4). The 30-day readmission rate was $10.6 \%$ in TS participants $(n=236)$ vs $16.3 \%$ for usual care $(n=941)$ $(0.65 ; 95 \%$ CI, 0.44 to $0.97 ; p=0.03$ ) (Table 3 ). CACE analyses showed an absolute $4.0 \%$ reduction in readmission rates in the 
Table 2 Primary and Secondary Outcomes (Intention to Treat)

\begin{tabular}{|c|c|c|c|}
\hline & $\begin{array}{l}\text { Transition services } \\
(n=935)\end{array}$ & $\begin{array}{l}\text { Usual care } \\
(n=941)\end{array}$ & $\begin{array}{l}\mathrm{RR}(95 \% \mathrm{CI}) \\
(p \text { value })\end{array}$ \\
\hline $\begin{array}{l}\text { 30-day all cause non-elective (CMS definition and inclusion } \\
\text { of observation patients and any CHS facility) }\end{array}$ & $142(15.2)$ & $153(16.3)$ & $\begin{array}{l}0.93(0.76-1.15) \\
(p=0.52)\end{array}$ \\
\hline Inpatient* & $124(13.3)$ & $128(13.6)$ & $\begin{array}{l}0.98(0.77-1.23) \\
(p=0.83)\end{array}$ \\
\hline Observation* & $21(2.3)$ & $33(3.5)$ & $\begin{array}{l}0.64(0.37-1.10) \\
(p=0.10)\end{array}$ \\
\hline Emergency Department visit within 30-days of discharge & $117(12.5)$ & $100(10.6)$ & $\begin{array}{l}1.10(0.95-1.28) \\
(p=0.20)\end{array}$ \\
\hline Length of stay - index admission, days, median (IQR) & $4.0(2.0)$ & $3.0(4.0)$ & $p=0.14$ \\
\hline Length of stay - initial readmission, days, median (IQR) & $\begin{array}{l}(n=142) \\
3.0(4.0)\end{array}$ & $\begin{array}{l}(n=153) \\
3.0(4.0)\end{array}$ & $p=0.52$ \\
\hline $\begin{array}{l}\text { 60-day all cause readmission rate (CMS definition and inclusion } \\
\text { of observation patients and any CHS facility) }\end{array}$ & $206(22.0)$ & $214(22.7)$ & $\begin{array}{l}0.97(0.82-1.15) \\
(p=0.71)\end{array}$ \\
\hline $\begin{array}{l}\text { 90-day all cause readmission rate (CMS definition and inclusion } \\
\text { of observation patients and any CHS facility) }\end{array}$ & $252(27.0)$ & $265(28.2)$ & $\begin{array}{l}0.96(0.83-1.11) \\
(p=0.56)\end{array}$ \\
\hline Intensive Care Unit stay on initial readmission & $\begin{array}{l}(n=142) \\
22(15.5)\end{array}$ & $\begin{array}{l}(n=153) \\
41(26.8)\end{array}$ & $\begin{array}{l}0.74(0.59-0.93) \\
(p=0.02)\end{array}$ \\
\hline 30-day readmission rate among patients with a primary & $(n=41)$ & $(n=48)$ & $1.17(0.51-2.67)$ \\
\hline diagnosis of congestive heart failure & $9(22.0)$ & $9(18.8)$ & $(p=0.71)$ \\
\hline 30-day readmission rate among patients with a primary & $(n=33)$ & $(n=24)$ & $0.44(0.12-1.65)$ \\
\hline diagnosis of pneumonia & $3(9.1)$ & $5(20.8)$ & $(p=0.26)$ \\
\hline 30-day readmission rate among patients with a primary & $(n=139)$ & $(n=129)$ & $0.49(0.24-0.97)$ \\
\hline diagnosis of sepsis & $11(7.9)$ & $21(16.3)$ & $(p=0.03)$ \\
\hline
\end{tabular}

Entries are $n(\%)$ unless otherwise indicated

*11 patients had both an inpatient and an observation encounter within 30 days of index encounter

intervention compared to the usual care $(-4.0 ; 95 \% \mathrm{CI},-17.0$ to $9.1 \% ; p=0.55)$.

\section{DISCUSSION}

When analyzed within a real-world, population health framework, referral of high-risk patients to a transitions program did not lead to reductions in 30-day readmissions. These study results may not be surprising in the context of fairly static national readmission trends. Rather, the results provide RCT evidence highlighting the difficulty of moving a populationbased metric within a complex healthcare environment.

Pre-specified subgroup ITT analyses did show a decrease in readmissions for patients discharged with sepsis and a reduction in ICU stays for readmitted patients. In light of the low partici- pation rate and potential for multiplicity, these results may be explained by chance alone and should be subjected to further examination. Alternatively, the improvements might be explained by the proactive monitoring and interventions offered by TS to those who participated, resulting in hospital referral prior to requiring ICU care and addressing preventable infectionrelated post-discharge issues.

To isolate the effect of participation in TS, we performed PP and CACE analyses, which provided mixed results. The PP analysis demonstrated a significant reduction in readmissions for TS participants; but as a consequence of neither preserving randomization, nor adjusting for the resultant confounding, PP analyses introduce inherent bias. In contrast, the CACE method incorporated the chance of being a complier (participant) and did not show a significant difference. While RCTs of population health interventions are challenging to conduct, these

Table 3 Primary and Secondary Outcomes (Per Protocol)

\begin{tabular}{|c|c|c|c|c|}
\hline & $\begin{array}{l}\text { Transition services- } \\
\text { attended } \\
(n=236)\end{array}$ & $\begin{array}{l}\text { Transition services- } \\
\text { did not attend } \\
(n=699)\end{array}$ & $\begin{array}{l}\text { Usual care } \\
(n=941)\end{array}$ & $\begin{array}{l}\text { RR }(95 \% \text { CI }) \\
(p \text { value }) \\
\text { TS attended vs. } \\
\text { usual care }\end{array}$ \\
\hline $\begin{array}{l}\text { 30-day all cause readmission (CMS definition and inclusion } \\
\text { of observation patients and any CHS facility) }\end{array}$ & $25(10.6)$ & $117(16.7)$ & $153(16.3)$ & $\begin{array}{l}0.65(0.44-0.97) \\
(p=0.03)\end{array}$ \\
\hline Emergency Department visit within 30 days of discharge & $28(11.9)$ & $89(12.7)$ & $100(10.6)$ & $\begin{array}{l}1.03(0.93-1.13) \\
(p=0.59)\end{array}$ \\
\hline Length of stay - index admission, days, median (IQR) & $4.0(2.0)$ & $4.0(3.0)$ & $3.0(4.0)$ & $p=0.65$ \\
\hline Length of stay - initial readmission, days, median (IQR) & $\begin{array}{l}(n=25) \\
4.0(4.0)\end{array}$ & $\begin{array}{l}(n=117) \\
3.0(4.0)\end{array}$ & $\begin{array}{l}(n=153) \\
3.0(4.0)\end{array}$ & $p=0.82$ \\
\hline $\begin{array}{l}\text { 60-day all cause readmission rate (CMS definition and } \\
\text { inclusion of observation patients and any CHS facility) }\end{array}$ & $43(18.2)$ & $163(23.3)$ & $214(22.7)$ & $\begin{array}{l}0.80(0.60-1.08) \\
(p=0.13)\end{array}$ \\
\hline $\begin{array}{l}\text { 90-day all cause readmission rate } \\
\text { (CMS definition and inclusion of observation patients and } \\
\text { any CHS facility) }\end{array}$ & $57(24.2)$ & $195(27.9)$ & $265(28.2)$ & $\begin{array}{l}0.86(0.67-1.10) \\
(p=0.22)\end{array}$ \\
\hline
\end{tabular}

Entries are $n(\%)$ unless otherwise indicated 
discrepancies between ITT, PP, and CACE analyses serve as a contemporary reminder to cautiously interpret conclusions from studies that rely on less rigorous methods.

This is the first US study to prospectively evaluate a complex transition intervention's effect on readmissions in a real-world setting. Our results align closely with similar international studies. In Canada, Dhalla et al. showed a non-significant absolute decrease of $3.4 \%$ in readmissions for patients attending a virtual post-discharge ward. That study only included interested, participating patients; thus, results may align more closely with our CACE analysis showing an absolute, non-significant reduction of 4\%. In Singapore, Low et al. found a virtual integrated practice unit resulted in a significant 33\% reduction in 30-day readmission rates. Low et al. also evaluated an engaged and interested population excluding $75 \%$ due to eligibility and an additional $30 \%$ of eligible patients declining participation. We found approximately the same reduction in readmissions (35\%) for a similarly engaged population as reflected in the PP analysis. The discordance between our ITT primary outcome is at least in part explained by the more pragmatic nature of our hypothesis and study design.

This study has several implications for health systems seeking to improve readmission rates. First, efforts to reduce readmissions are challenging to implement in the real world where a system is accountable for an entire population, not merely patients who are engaged and who participate. Effective readmission-reducing interventions will need to embrace flexibility. For example, participants who declined often expressed preference to follow-up with their Primary Care Providers (PCPs) or concern about travel time. Adaptations might include virtual integration of TS into primary care or an increased emphasis on virtual home visit availability. Second, bridging transition interventions connect with patients prior to discharge within a fast-moving, complex, hospital environment. Making this in-person connection is challenging and reflected by one third of patients in the TS referral group not being contacted prior to discharge for reasons such as being away from their room for studies, not feeling well enough to talk, or being discharged before the navigator arrived. Health systems implementing bridging interventions may need to increase navigator staffing or expand navigator reach with virtual technology.

Our study has several limitations. First, the study's power suffered from a very high crossover to usual care (74.8\%), resulting in significant attenuation of outcomes, but providing an accurate reflection of real-world effectiveness. The lower than expected readmission rate in usual care $(16.3 \%$ vs $20 \%)$ is likely due to exclusion of known high-risk patients (SNF, drug dependence, psychosis, and active malignancy), but may also be due to other contemporaneous efforts to improve readmissions. Second, as a consequence of the study's pragmatic design, we would expect missing data from utilization outside the sponsoring system; however, missing data should be non-differential across groups and would be reflected in lower overall readmission rates. Third, the reapplication of exclusion criteria at discharge was necessary to allow the intervention to begin early in a patient's hospitalization, opening up the possibility for selection bias; but characteristics were balanced across groups. Fourth, the required in-person introduction to TS resulted in a significant proportion of crossovers to usual care but demonstrates an accurate estimation of the potential reach of an in-person navigator in the acute setting. Fifth, this study was not powered to determine the intervention's effectiveness in subgroups, thus these analyses should be interpreted with caution. Lastly, generalizability may be limited as the intervention included a single care team and healthcare system, though overall represents a diverse population with minimal exclusions applied.

\section{CONCLUSIONS}

For healthcare systems seeking guidance on how to avoid 30-day readmission penalties, the evidence remains mixed. While this study's results are inconclusive, they highlight the need for future research on novel approaches designed to address the reality of ubiquitous, low participation rates in real-world implementation. Furthermore, as healthcare spending continues to climb, the discrepant outcomes from the ITT, PP, and CACE analyses emphasize the need for population health innovations to be subjected to rigorous, pragmatic evaluations, lest they add cost without additional benefits to patients.

Corresponding Author: Andrew McWilliams, MD,MPH; Carolinas Hospitalist Group, Center for Outcomes Research and Evaluation, Carolinas Health Care System, 1540 Garden Terrace, Charlotte, NC, 28203, USA (e-mail: andrew.mcwilliams@carolinashealthcare.org).

\section{Primary Funding Source Carolinas HealthCare System}

Compliance with Ethical Standards The trial was approved by the Carolinas HealthCare System Institutional Review Board and granted a waiver for patient consent (reference number 01-15-10E).

Conflict of Interest: $A M$ has received funding support for research from AstraZeneca, Amylin Pharmaceuticals, and is a cofounder of iEnroll, LLC. No other authors report any potential conflicts of interest.

\section{REFERENCES}

1. Mendelson A KK, Damberg C, Low A, Motuapuaka M, Freeman M, O'Neil M, Relevo R, Kansagara D. The effects of pay-for-performance programs on health, health care use, and processes of care. Ann Intern Med.. 2017;166(5):341-53.

2. Zuckerman RB, Sheingold SH, Orav EJ, Ruhter J, Epstein AM. Readmissions, observation, and the hospital readmissions reduction program. N Engl J Med. 2016;374(16):1543-51. https://doi.org/10. 1056/NEJMsa 1513024

3. Mellor J, Daly M, Smith M. Does it pay to penalize hospitals for excess readmissions? Intended and unintended consequences of medicare's hospital readmissions reductions program. Health Econ. 2017;26(8):1037-51. https://doi.org/10.1002/hec.3382.

4. Carey K, Lin MY. Readmissions to new york hospitals fell for three target conditions from 2008 to 2012, consistent with medicare goals. Health Aff (Millwood). 2015;34(6):978-85. https://doi.org/10.1377/hlthaff.2014.1408.

5. Rennke S, Nguyen OK, Shoeb MH, Magan Y, Wachter RM, Ranji SR. Hospital-initiated transitional care interventions as a patient safety strategy: a systematic review. Ann Intern Med. 2013;158(5 Pt 2):43340. https://doi.org/10.7326/0003-4819-158-5-201303051-00011.

6. Kansagara D, Chiovaro JC, Kagen D, Jencks S, Rhyne K, O'Neil M, et al. So many options, where do we start? An overview of the care transitions literature. J Hosp Med. 2015. https://doi.org/10.1002/jhm.2502. 
7. Kripalani S, Theobald CN, Anctil B, Vasilevskis EE. Reducing hospital readmission rates: current strategies and future directions. Annu Rev Med. 2014;65:471-85.https://doi.org/10.1146/annurev-med-022613-090415.

8. Hansen LO, Young RS, Hinami $\mathbf{K}$, Leung A, Williams MV. Interventions to reduce 30-day rehospitalization: a systematic review. Ann Intern Med. 2011;155(8):520-8. https://doi.org/10.7326/0003-4819-155-8201110180-00008.

9. Leppin AL, Gionfriddo MR, Kessler M, Brito JP, Mair FS, Gallacher K, et al. Preventing 30-day hospital readmissions: a systematic review and meta-analysis of randomized trials. JAMA Intern Med. 2014;174(7):1095107. https://doi.org/10.1001/jamainternmed.2014.1608.

10. Low LL, Tan SY, Ng MJ, Tay WY, Ng LB, Balasubramaniam K, et al. Applying the Integrated Practice Unit Concept to a Modified Virtual Ward Model of Care for Patients at Highest Risk of Readmission: A Randomized Controlled Trial. PLoS One. 2017;12(1):e0168757. https://doi.org/10. 1371/journal.pone.0168757.

11. Cavanaugh JJ, Jones CD, Embree G, Tsai K, Miller T, Shilliday BB, et al. Implementation Science Workshop: primary care-based multidisciplinary readmission prevention program. J Gen Intern Med. 2014;29(5):798-804. https://doi.org/10.1007/s11606-014-2819-8.

12. Dhalla IA, O'Brien T, Morra D, Thorpe KE, Wong BM, Mehta R, et al. Effect of a postdischarge virtual ward on readmission or death for highrisk patients: a randomized clinical trial. JAMA. 2014;312(13):1305-12. https://doi.org/10.1001/jama.2014.11492.

13. Thorpe KE, Zwarenstein M, Oxman AD, Treweek S, Furberg CD, Altman DG, et al. A pragmatic-explanatory continuum indicator summary (PRECIS): a tool to help trial designers. CMAJ. 2009;180(10):E4757. https://doi.org/10.1503/cmaj.090523.
14. Loudon $\mathbf{K}$, Treweek S, Sullivan F, Donnan P, Thorpe KE, Zwarenstein M. The PRECIS-2 tool: designing trials that are fit for purpose. BMJ. 2015;350:h2147. https://doi.org/10.1136/bmj.h2147.

15. School HB. Integrated Practice Unit. http://www.isc.hbs.edu/healthcare/vbhcd/Pages/integrated-practice-units.aspx. Accessed July 2018.

16. McWilliams A, Roberge J, Moore CG, Ashby A, Rossman W, Murphy S, et al. Aiming to Improve Readmissions Through InteGrated Hospital Transitions (AIRTIGHT): study protocol for a randomized controlled trial. Trials. 2016;17(1):603. https://doi.org/10.1186/s13063-016-1725-2.

17. Coleman EA, Parry C, Chalmers S, Min SJ. The care transitions intervention: results of a randomized controlled trial. Arch Intern Med. 2006;166(17):1822-8. https://doi.org/10.1001/archinte.166.17.1822.

18. Jack BW, Chetty VK, Anthony D, Greenwald JL, Sanchez GM, Johnson AE, et al. A reengineered hospital discharge program to decrease rehospitalization: a randomized trial. Ann Intern Med. 2009;150(3):178-87.

19. Caplan GA, Sulaiman NS, Mangin DA, Aimonino Ricauda N, Wilson AD, Barclay L. A meta-analysis of "hospital in the home". Med J Aust. 2012; 197(9):512-9.

20. SAS Enterprise Guide 6.1 for Windows on platform 9.4.1,. Cary, NC: SAS Institute Inc.

21. Hernan MA, Hernandez-Diaz S. Beyond the intention-to-treat in comparative effectiveness research. Clin Trials. 2012;9(1):48-55. https://doi.org/10.1177/1740774511420743.

22. Dunn G, Maracy M. Tomenson Estimating treatment effects from randomized clinical trials with noncompliance and loss to follow-up: the role of instrumental variable methods. Stat Methods Med Res. 2005; 14(4):369-95.

\section{APPENDIX}

Table 4 Patient Characteristics for Participants and Non-participants

\begin{tabular}{|c|c|c|c|}
\hline & $\begin{array}{l}\text { Participated in TS } \\
(n=236)\end{array}$ & $\begin{array}{l}\text { Did not participate in TS } \\
(n=699)\end{array}$ & $p$ value \\
\hline Male, $n(\%)$ & $114(48.3)$ & $343(49.1)$ & 0.84 \\
\hline Hispanic, $n(\%)$ & $\begin{array}{l}(n=224) \\
14(6.3)\end{array}$ & $\begin{array}{l}(n=645) \\
47(7.3)\end{array}$ & 0.60 \\
\hline Race, $n(\%)$ & $(n=231)$ & $(n=685)$ & \\
\hline American Indian/Alaska Native & $0(0)$ & $1(0.2)$ & \\
\hline Asian & $1(0.4)$ & $14(2.0)$ & \\
\hline Black & $116(50.2)$ & $244(35.6)$ & \\
\hline White & $98(42.4)$ & $365(53.3)$ & \\
\hline Multi & $1(0.4)$ & $1(0.2)$ & \\
\hline Other & $15(6.5)$ & $60(8.8)$ & \\
\hline Age, mean (SD) & $54.8(17.1)$ & $59.5(17.7)$ & 0.0004 \\
\hline Primary diagnosis, $n(\%)$ & $(n=233)$ & $(n=671)$ & \\
\hline Complications of pregnancy; childbirth; and the puerperium & $0(0)$ & $4(0.6)$ & \\
\hline Congenital anomalies & $0(0)$ & $2(0.3)$ & \\
\hline Diseases of the blood and blood-forming organs & $3(1.3)$ & $13(1.9)$ & \\
\hline Diseases of the circulatory system & $45(19.3)$ & $158(23.5)$ & \\
\hline Diseases of the digestive system & $40(17.2)$ & $75(11.2)$ & \\
\hline Diseases of the genitourinary system & $19(8.2)$ & $26(3.9)$ & \\
\hline Diseases of the musculoskeletal system and connective tissue & $13(5.6)$ & $18(2.7)$ & \\
\hline Diseases of the nervous system and sense organs & $7(3.0)$ & $33(4.9)$ & \\
\hline Diseases of the respiratory system & $35(15.0)$ & $84(12.5)$ & \\
\hline Diseases of the skin and subcutaneous tissue & $9(3.9)$ & $20(3.0)$ & \\
\hline Endocrine; nutritional; and metabolic diseases and immunity disorders & $19(8.2)$ & $55(8.2)$ & \\
\hline Infectious and parasitic diseases & $22(9.4)$ & $70(10.4)$ & \\
\hline Injury and poisoning & $9(3.9)$ & $50(7.5)$ & \\
\hline Mental illness & $4(1.7)$ & $6(0.9)$ & \\
\hline Neoplasms & $3(1.3)$ & $47(7.0)$ & \\
\hline Residual codes; unclassified; all E codes [259. and 260.] & $0(0)$ & $1(0.1)$ & \\
\hline Symptoms; signs; and ill-defined conditions and factors influencing health status & $5(2.1)$ & $9(1.3)$ & \\
\hline \multicolumn{4}{|l|}{ Comorbidities, $n(\%)$} \\
\hline Chronic obstructive pulmonary disease & $43(18.2)$ & $154(22.0)$ & 0.21 \\
\hline Congestive heart failure & $61(25.9)$ & $164(23.5)$ & 0.46 \\
\hline Diabetes & $97(41.1)$ & $262(37.5)$ & 0.32 \\
\hline End-stage renal disease & $25(10.6)$ & $87(12.5)$ & 0.45 \\
\hline Health insurance, $n(\%)$ & $(n=236)$ & $(n=688)$ & 0.007 \\
\hline Commercial/private & $62(26.3)$ & $203(29.5)$ & \\
\hline Medicaid & $43(18.2)$ & $91(13.2)$ & \\
\hline Medicare & $88(37.3)$ & $308(44.8)$ & \\
\hline Self pay & $38(16.1)$ & $64(9.3)$ & \\
\hline Other & $5(2.1)$ & $22(3.2)$ & \\
\hline
\end{tabular}

